\title{
Die richterliche Bewertung von Ehrenmorden in Deutschland
}

Eine empirische Analyse der Sanktionspraxis im Zeitraum 1996 bis 2005

\author{
von Julia Kasselt und Dietrich Oberwittler
}

\section{Zusammenfassung}

Der Artikel beschäftigt sich mit der Frage, wie deutsche Schwurgerichtskammern das Phänomen der sogenannten Ehrenmorde juristisch einordnen. Auf der Basis von Strafaktenanalysen wird überprüft, ob und inwieweit die Landgerichte sich an der einschlägigen Rechtsprechung des Bundesgerichtshofs orientieren und die Täter wegen Mordes aus niedrigen Beweggründen nach $\$ 211$ StGB verurteilen. Zudem wird vergleichend die Strafzumessungspraxis in einer Stichprobe von Partnertötungsfällen untersucht. Die Analysen belegen, dass Ehrenmörder von deutschen Schwurkammern im untersuchten Zeitraum von 1996 bis 2005 sehr unterschiedlich sanktioniert wurden, wobei sich ab 2002 ein signifikanter Trend zu höheren Strafen, insbesondere zu mehr lebenslangen Freiheitsstrafen, zeigt. Der quantitative Vergleich mit den Partnertötungen illustriert, dass diese insgesamt milder beurteilt werden als Ehrenmorde. Auffällig ist, dass die Partnertötungen über den gesamten Untersuchungszeitraum hinweg gleichmäßig bestraft wurden. Der Anstieg der Strafhöhen in den Ehrenmordurteilen ab 2002 ist somit wohl nicht auf einen generellen punitiven Trend bei der Sanktionierung von Tötungsdelikten in Deutschland, sondern vielmehr auf einen Wandel in der Rechtsprechung des Bundesgerichtshofs im Hinblick auf die Bestrafung von Tötungsdelikten mit Ehrhintergrund zurückzuführen.

Schlüsselwörter: Ehrenmord, Strafzumessung, Mord, Totschlag, niedrige Beweggründe

\section{The Judicial Interpretation of Honour Killings in Germany}

An empirical analysis of the sentencing practice between 1996 and 2005

\section{Abstract}

The article discusses the question of how German criminal courts interpret the phenomenon of honour killings. Based on analyses of criminal case records, it examines whether and to what extent district courts follow the jurisprudence of the German Federal Court of Justice (Bundesgerichtshof) by convicting the perpetrators of murder for base motives according to $\mathbb{} 211$ of the German Criminal Code (StGB). Furthermore, a comparative sentencing analysis from a sample of cases involving intimate partner killings is conducted. The findings demonstrate that German criminal courts punished the perpetrators of honour killings very differently in the examined timeframe between 1996 and 2005. From 2002 onwards, significantly harsher sentences, particularly more life sentences, were imposed. The quantitative comparison of the two homicide case samples shows that intimate partner killings are punished more leniently. A remarkable result of the study is that no positive or negative trend was proven for the intimate partner killings in the examined timeframe. This implies that there is no general tendency to harsher sentences in homicide cases in Germany. Instead, the increase in sentencing in honour killing cases is probably attributable to a change in the jurisprudence of the German Federal Court of Justice regarding the penalisation of those cases.

Keywords: Honour killing, sentencing, homicide, murder, manslaughter

\section{Einleitung}

Die Ermordung der Deutschtürkin Hatun Sürücü in Berlin im Februar 2005 löste in Deutschland eine große öffentliche Aufmerksamkeit für die Thematik der sogenannten Ehrenmorde aus, die bis heute andauert. Die junge Frau wurde auf offener Straße von mindestens einem ihrer Brüder erschossen, weil sie durch ihren »westlichen«, emanzipierten 
Lebensstil aus Sicht ihrer Angehörigen die Familienehre verletzt hatte. ${ }^{1}$ Aus hiesiger Perspektive erscheint die Tötung eines Familienmitgliedes aufgrund kollektiver Ehrnormen archaisch und grausam, denn die westlichen Wertvorstellungen unterscheiden sich deutlich von dem Ehrverständnis, auf welches sich die Ehrenmörder berufen. ${ }^{2}$ Die Taten werden daher häufig im Rahmen der in regelmäßigen Abständen aufflackernden Integrationsdebatte als Beleg für die Integrationsdefizite von vorrangig türkischen oder generell muslimischen Migranten sowie für die Existenz angeblicher »Parallelgesellschaften« dieser Minderheiten herangezogen (Oberwittler E Kasselt 2011, 1 f.; Oberwittler E Kasselt 2014). ${ }^{3}$

Auch die Problematik der strafrechtlichen Ahndung der Ehrenmorde steht im Fokus der öffentlichen Debatte. So löste die Äußerung Hassemers, bei der Bestrafung von Ehrenmördern müsse auch die Sozialisation der Täter berücksichtigt und demgemäß sollten auch Strafmilderungen in Betracht gezogen werden (Hassemer im Interview mit Darnstädt und Stoldt, 13.05.2009), bei Frauenrechtsorganisationen und auf Regierungsebene Entrüstung aus. Hassemers Kritiker argumentierten, Strafmilderungen für Ehrenmörder würden der Entstehung von »Parallelgesellschaften « Vorschub leisten und die Präventionsbemühungen von Menschenrechtsorganisationen hinsichtlich solcher Taten konterkarieren. ${ }^{4}$

Die Diskussion verdeutlicht das Dilemma, mit dem sich die deutsche Justiz bei der strafrechtlichen Bewertung der Ehrenmorde konfrontiert sieht: Sollten die Taten ausschließlich auf Grundlage der deutschen Wertvorstellungen bewertet werden, um dem Gerechtigkeitsund Strafbedürfnis der deutschen Mehrheitsgesellschaft zu entsprechen und Migranten zu verdeutlichen, dass sie sich an die deutsche Rechtsordnung zu halten haben? Oder erfordert das Recht des Täters auf eine individuelle, schuldangemessene Strafe eine strafmildernde Berücksichtigung seiner Sozialisation in einem fremden Kulturkreis? Werden die Taten milder oder strenger bestraft als vergleichbare, durch deutsche Täter begangene Tötungsdelikte?

In der hier vorgestellten Studie wurde auf Basis von Strafakten untersucht, wie die Schwurkammern der Landgerichte dieses Dilemma zu lösen versuchen. Nachfolgend wird zuerst die der Untersuchung zugrunde liegende Arbeitsdefinition des Terminus »Ehrenmord « näher beleuchtet. Anschließend erfolgt ein Abriss zur Rechtsprechung des Bundesgerichtshofs (BGH) in Ehrenmordfällen sowie anderen Fällen kulturell geprägter (Tötungs-)Delikte. Sodann werden darauf aufbauend die Fragestellung und die Ziele der Studie skizziert. Im Anschluss daran wird das methodische Vorgehen dargelegt und danach werden ausgewählte Ergebnisse der empirischen Studie vorgestellt und diskutiert. Abschließend werden die Befunde zusammengefasst und es erfolgt ein Ausblick auf sich anschließende Forschungsfragen.

\section{Arbeitsdefinition "Ebrenmord"}

In der mittlerweile mehrere Jahre andauernden öffentlichen und akademischen Auseinandersetzung mit dem Phänomen der Ehrenmorde hat sich noch keine allgemeingültige Definition des Terminus »Ehrenmord « beziehungsweise seines englischen Synonyms »hono $(u) r$ killing « herauskristallisiert. Für die hier vorgestellte Studie wurde die von Oberwittler und Kasselt (2011) entwickelte Arbeitsdefinition zugrunde gelegt (vgl. hierzu sowie zu den nachfolgenden Ausführungen Oberwittler $\mathcal{E}$ Kasselt 2011, 22 ff.). Diese ist eher weit angelegt, um alle Schattierungen und Randbereiche des Phänomens zu erfassen. Zusätzlich wurden auf

1 Vgl. zu dem Fall detailliert Deiß E Goll 2011.

2 Vgl. zu diesem Ehrkonzept ausführlich Cöster 2009, 42 ff.

3 Zur Diskussion um mögliche Parallelgesellschaften vgl. Schiffaner 2008.

4 Vgl. die Pressemitteilung der Bundesregierung vom 14.05.2009 sowie die Stellungnahme von TERRE DES FEMMES vom 20.05.2009. 
Grundlage der Erkenntnisse aus der Literatur sowie dem der Studie zugrunde liegenden empirischen Fallmaterial Subtypen gebildet, um die verschiedenen Ehrenmordformen voneinander abzugrenzen. Die Hauptdefinition lautet:

Ehrenmorde sind vorsätzlich begangene, versuchte oder vollendete Tötungsdelikte, die im Kontext patriarchalisch geprägter Familienverbände oder Gesellschaften vorrangig von Männern an Frauen verübt werden, um die aus Tätersicht verletzte Ehre der Familie oder des Mannes wiederherzustellen. Die Verletzung der Ehre erfolgt durch einen wahrgenommenen Verstoß einer Frau gegen auf die weibliche Sexualität bezogene Verhaltensnormen.

Aus dieser Definition sind folgende Merkmale von Ehrenmorden abzuleiten:

1. (Ein) Tatmotiv ist die Wiederherstellung der nach Ansicht des Täters beschädigten Ehre der Familie oder eines Mannes.

2. Die Ehrverletzung wurde durch den Verstoß einer Frau gegen bestimmte Verhaltensnormen ausgelöst, die sich im weitesten Sinne auf die sexuelle Reinheit der Frau beziehen. Als ehrverletzend werden von den Tätern insbesondere die tatsächliche oder vermeintliche Untreue sowie die Aufnahme einer vorehelichen Intimbeziehung interpretiert (vgl. u.a. Tellenbach 2003, 77). Mögliche Tatanlässe sind zudem die Verweigerung einer arrangierten Ehe sowie die von der Frau initiierte Trennung vom Ehemann (vgl. Kaspersson 2009, 23, 26; Khan 2012, 81). Des Weiteren kann, wie im Fall Sürüc̈̈, auch ein als zu westlich empfundener Lebens- und Kleidungsstil einer jungen Frau zu einem Ehrenmord führen.

3. Sowohl Frauen als auch Männer können Opfer eines Ehrenmordes werden. Bei den männlichen Opfern handelt es sich meistens um die als illegitim empfundenen Partner eines weiblichen Familienmitgliedes, die entweder zusätzlich oder anstelle der Frau getötet werden.

4. Als Täter kommen auch Frauen in Betracht. Zwar treten Frauen nur in absoluten Ausnahmefällen als direkte oder gar Einzeltäterinnen auf, allerdings sind sie nicht selten als Anstifterinnen oder Gehilfinnen an den Taten beteiligt.

5. Zentrales Element der Ehrenmorde ist die Bedeutung des (familiären) Kollektivs. Ehrenmorde entspringen häufig einem gemeinschaftlichen Tatentschluss des »Familienrats «, mitunter sind mehrere Familienmitglieder aktiv an der Tat beteiligt. Aber auch bei Ehrenmorden, die von einem Einzeltäter begangen wurden, spielt das Kollektiv eine bedeutende Rolle: Die Taten werden zumindest von Teilen des Verwandten- und/oder Freundeskreises des Täters und/oder Opfers gebilligt oder gar aktiv oder passiv unterstützt, weil das Kollektiv dieselben Ehrnormen vertritt wie der Täter. Häufig wird auf den Täter von Verwandten, Freunden und/oder Bekannten Druck ausgeübt, seine oder die Familienehre wiederherzustellen, wenn die »Verfehlung « des späteren Tatopfers bekannt geworden ist. Deutlich wird die Bedeutung des Kollektivs auch in Fällen, in denen der Täter das seiner Ansicht nach ehrverletzende Verhalten des späteren Tatopfers toleriert, solange es noch nicht über die Grenzen der (Kern-)Familie hinaus bekannt geworden ist. Erst das Gerede der Bekannten und Freunde über die »Schande« führt dann beim Täter zum Tötungsentschluss.

Die zentrale Rolle des Familienkollektivs verweist auf die sozialen Kontexte, in denen Ehrenmorde geschehen (vgl. hierzu Oberwittler $\mathcal{E}$ Kasselt 2011, 28 ff.): in agrarischen Ländern mit wenig fruchtbaren Böden (vor allem in Vorder- und Mittelasien sowie Nordafrika), mit schwachen staatlichen Strukturen und starken Familienverbänden, die das wirtschaftliche und soziale Rückgrat der Gesellschaft bilden, sowie bei Migranten in westlichen Ländern, die aus solchen ländlichen Strukturen stammen. Frauen wird in diesen Gesellschaften traditionell eine passive und untergeordnete Rolle zugewiesen. Ein davon abweichendes, selbstbestimmtes Verhalten von Frauen wird dementsprechend als Verletzung der kollektiven Ehre der ganzen Familie verstanden, die im Extremfall nur durch ihre Tötung gutgemacht werden kann. »Ehre« ist in diesem gesellschaftlichen Kontext, in dem Familienclans gegen- 
einander um Land, Wasser und Vieh kämpfen, »the reputation for being willing and able to carry out violence (Nisbett \& Cohen 1996, 89). Diese in Jahrtausenden gewachsenen Normen bleiben für das Verständnis der von Migranten in westlichen Gesellschaften begangenen Ehrenmorde relevant, auch wenn die Migrationssituation der Problemkonstellation eine zusätzliche Dimension hinzufügt.

Da das Phänomen der Ehrenmorde verschiedene Differenzierungen und Überschneidungen mit anderen Deliktsformen aufweist, wird hier zwischen vier Subtypen unterschieden.

Ein Ehrenmord im engeren Sinne ist die Tötung einer Frau durch ihre Blutsverwandten zur Wiederherstellung der kollektiven Familienehre. Die Opfer sind entweder unverheiratete junge Frauen, deren Verhalten gegen die Ehrnormen verstößt oder verheiratete Frauen, die aber dennoch nicht von ihrem Ehemann, sondern nach den archaischen Regeln des Stammesrechts von ihrer Herkunftsfamilie getötet werden. Es können jedoch weitere, nicht blutsverwandte Personen als Täter oder Opfer in diese Fälle involviert sein. Besonders häufig wird der von der Herkunftsfamilie einer jungen Frau unerwünschte Intimpartner getötet.

Grenzfälle zur Partnertötung sind Tötungsdelikte, in deren Zentrum die Täter-OpferKonstellation Intimpartnerschaft steht. In der Regel handelt es sich um Fälle, in denen die von der Frau ausgehende Trennung beziehungsweise die Trennungsabsicht oder (vermutete) Untreue der Frau den Anlass für die gewaltsame Reaktion des (Ex-)Partners bildet. Falls die Partner zugleich Blutsverwandte sind, was vor allem im Rahmen der sogenannten Parallelcousinen-Heirat vorkommt, geht hier die Partnerschaftsbeziehung vor. Im Unterschied zu reinen Partnertötungen liegt diesen Grenzfällen - wie bei den Ehrenmorden im engeren Sinne - eine kollektive Ehrauffassung des Täters zugrunde und die Tat wird in der Regel vom sozialen Umfeld des Täters aktiv unterstützt oder zumindest gebilligt. Als Grenzfälle zur Partnertötung wurden in der hier vorgestellten Studie auch Fälle eingeordnet, bei denen - teilweise zusätzlich zur Frau - der Täter seinen (vermeintlichen) Nebenbuhler, also den (angeblichen) Liebhaber oder neuen Partner der Frau, getötet hat. Der Grund für diese Zuordnung liegt darin, dass der tatauslösende Konflikt in der Intimpartnerschaft begründet ist und sich die Tatvorgeschichte in Fällen, in denen nur der Nebenbuhler getötet wurde, kaum von denen unterscheidet, in denen der Täter seine (Ex-)Partnerin getötet hat. So kommt es im Vorfeld dieser Taten beispielsweise häufig zu Todesdrohungen gegenüber der Frau, auch wenn später nicht sie, sondern der Nebenbuhler getötet wird. Zudem steht - wie bei den Partnertötungen - der Aspekt der Eifersucht im Vordergrund.

Grenzfälle zur Blutrache sind Tötungsdelikte, bei denen eine verwandtschaftliche Beziehung zwischen einem Täter und einem direkten oder indirekten Opfer vorliegt, die aber zugleich typische Merkmale der Blutrache aufweisen, die also im Kontext von Konflikten zwischen zwei Familienverbänden verübt werden und/oder den Charakter der Vergeltung einer Ehrverletzung tragen. ${ }^{5}$ Als »indirektes Opfer « werden weibliche Familienangehörige der Täter eingeordnet, deren Verhalten als Ehrverstoß betrachtet wird, die aber selbst nicht angegriffen wurden. Indirekte Opfer sind dementsprechend Frauen, deren von der Familie nicht akzeptierter Intimpartner getötet oder angegriffen wurde. Als Grenzfälle zur Blutrache wurden in dieser Studie vorrangig Taten eingeordnet, bei denen nur der unerwünschte Intimpartner eines weiblichen Familienmitgliedes getötet wurde. Da das Opfer aus einem anderen Familienverband stammt, weisen solche Taten Parallelen zur Blutrache auf. Der tatauslösende Konflikt besteht bei solchen Taten aber, wie bei den Ehrenmorden im engeren Sinne, vorrangig zwischen der Familie und der jungen Frau: Diese wird oft über einen län-

5 Vgl. zum Phänomen der Blutrache ausführlich u.a. Elster 1990; Singer 1994. 
geren Zeitraum hinweg zur Trennung gedrängt und häufig auch von den späteren Tätern bedroht.

Als reine Blutrachetat - und damit nicht als Ehrenmord - wurden hingegen Tötungen des Vergewaltigers einer Frau durch einen nicht zur eigenen Familie gehörenden Mann eingeordnet. Wenn der Vergewaltiger allerdings aus derselben Familie stammt, wurde die Tat ebenfalls als Blutrachegrenzfall eingeordnet und damit ins Ehrenmordsample einbezogen, weil die Tötung dann eben nicht außerhalb des eigenen Familienverbandes erfolgte und damit ein zwingendes Kriterium der Blutrachetötung nicht erfüllt war. Zudem folgten die Täter in den als Grenzfälle zur Blutrache eingeordneten Taten bei der Ausführung der Tötung nicht den formalen Regeln des Blutracherituals.

Sonstige Fälle und Mischtypen sind Fälle, die keinem der genannten Typen entsprechen, aber dennoch einen Ehrbezug aufweisen, oder die Charakteristika mehrerer der drei Typen miteinander verbinden und sich daher nicht eindeutig einem Typus zuordnen lassen.

\section{Höchstrichterliche Rechtsprechung zu Ehrenmorden und anderen in Deutschland begangenen kulturell geprägten Tötungsdelikten}

Die Rechtsprechung des BGH hinsichtlich der strafrechtlichen Einordnung von Tötungsdelikten mit ehrbezogenem Tathintergrund fokussiert sich auf die Frage, ob das Ehrmotiv als niedriger Beweggrund i.S.v. $\ 211$ StGB zu bewerten ist und dementsprechend eine Verurteilung wegen Mordes zu erfolgen hat. Der BGH hat seine Position in Bezug auf diese Thematik im Verlauf der letzten Jahrzehnte mehrmals geändert, zu unterscheiden sind dabei drei Phasen (vgl. u.a. Saliger 2003, 22; Baumeister 2007, 141; Erbil 2008, 178; Valerius 2011, 72). Die Änderungen beziehen sich auf die Frage, ob die heimatlichen Wertvorstellungen des Täters schon im Rahmen der Abwägungen zum objektiven Vorliegen eines niedrigen Beweggrundes oder erst auf subjektiver Ebene zu berücksichtigen sind.

In den 1960er und 1970er Jahren bewertete der BGH ehrbezogene Tatmotive nicht als niedrige Beweggründe und damit nicht als Mordmerkmale i.S.v. \$211 StGB. Bei der Begründung dieser Ansicht nahm der BGH Bezug auf die eigene Rechtsprechung zur Beachtung von Persönlichkeitsmängeln im Rahmen der sittlichen Bewertung einer Tat und betonte, dass diese Grundsätze nicht nur für psychopathologische Persönlichkeiten, sondern »erst recht « auch für Ausländer gelten müssten, die eine aus hiesiger Sicht besonders verwerfliche Tat begangen haben, weil sie in den "Anschauungen und Vorstellungen ihrer Heimat befangen « waren (BGH GA 1967, 244). Da der BGH das Vorliegen eines niedrigen Beweggrundes in dieser Rechtsprechungsphase mit dem Fehlen des subjektiven Elements des Mordmerkmals der niedrigen Beweggründe begründete, wird diese auch als »(erste) subjektive Phase « bezeichnet (u.a. von Saliger 2003, 67; Baumeister 2007, 141 und Çakir-Ceylan 2011, 227).

Ein Urteil des 5. Strafsenats des BGH aus dem Jahr 1979 leitete die zweite, sogenannte »objektive Phase« ein (vgl. Saliger 2003, 23; Baumeister 2007, 142; Agel 2013, 67). Der Senat führte in dieser Entscheidung aus, dass die heimatlichen Anschauungen und Wertvorstellungen von Tätern, die eine ehrbedingte Tötung begangen haben, schon im Rahmen der Abwägungen zum objektiven Vorliegen eines niedrigen Beweggrundes geprüft werden müssten (siehe BGH NJW 1980, 537). Die anderen Strafsenate des BGH folgten dieser Rechtsprechungslinie in mehreren Entscheidungen. Das letzte entsprechende Urteil stammt aus dem Jahr 1997: Der 4. Strafsenat bestätigte die Entscheidung der Vorinstanz, welche das objektive Vorliegen eines niedrigen Beweggrundes wegen der heimatlichen Wertvorstellungen des Angeklagten abgelehnt hatte (siehe BGH StV 1997, 566).

Die dritte, bis heute andauernde Rechtsprechungsphase begann mit einem Urteil des 2. Strafsenats, welches 1994, also drei Jahre vor der letzten Entscheidung des 4. Strafsenats aus der »objektiven Phase« fiel. Der Übergang von der zweiten zur dritten Phase verlief 
somit nicht trennscharf, sondern war durch widersprüchliche Urteile der verschiedenen Senate gekennzeichnet (vgl. Valerius 2011, S. 72; ähnlich auch Burmeister 2011, 73). Die Richter des 2. Senats entschieden, dass die Wiederherstellung der Ehre grundsätzlich als niedriger Beweggrund i.S.v. $\$ 211$ StGB zu bewerten ist, weil der Maßstab für die Bewertung eines Tatmotivs »den Vorstellungen der Rechtsgemeinschaft in der Bundesrepublik Deutschland zu entnehmen « sei »und nicht den Anschauungen einer Volksgruppe, die die sittlichen und rechtlichen Werte dieser Rechtsgemeinschaft nicht anerkennt « (BGH NStZ 1995, 79). Sie führten weiter aus, dass nur ausnahmsweise ein Ausschluss dieses Mordmerkmals auf subjektiver Ebene in Betracht komme, wenn der Täter nicht in der Lage war, sich von den heimatlichen Wertvorstellungen zu lösen (siehe BGH NStZ 1995, 79). Wegen der Verlagerung der Problematik auf die subjektive Ebene wird die dritte Phase auch »zweite subjektive Phase« genannt (vgl. u.a. Baumeister 2007, 142; Çakir-Ceylan 2011, 232; Agel 2013, 68). Im Jahr 2002 schloss der 5. Strafsenat sich der Linie des 2. Senats an und bekräftigte die Grundsatz-Ausnahme-Regelung hinsichtlich der Einstufung von ehrbezogenen Tötungsdelikten als Mord i.S.v. \211 StGB oder Totschlag i.S.v. $\mathbb{2} 212$ StGB (siehe BGH NStZ 2002, 369 f.). Diese Rechtsprechungslinie wurde seither in weiteren Entscheidungen des BGH bekräftigt (siehe BGH NStZ 2004, 332 f. und BGH NStZ 2006, 284 ff.).

Somit ist nach der aktuellen Rechtsprechung des BGH ein Ehrenmord in der Regel als Mord i.S.v. $\$ 211$ StGB einzustufen. Eine Verurteilung wegen Totschlags i.S.v. $\ 212$ StGB kann nur in Ausnahmefällen erfolgen, wenn der Täter nicht in der Lage war, sich von seinen heimatlichen Wertvorstellungen zu lösen. Der BGH billigt den Schwurkammern der Landgerichte mit dieser Rechtsprechung einen deutlichen Ermessensspielraum zu - insbesondere, da er nicht präzisiert hat, anhand welcher Kriterien die Schwurkammern die Intensität der Bindung eines Täters an seine heimatlichen Wertvorstellungen bewerten sollen.

Ein weiterer relevanter Aspekt im Hinblick auf die strafrechtliche Bewertung von Ehrenmorden ist die Frage, ob der kulturelle Tathintergrund im Rahmen der Strafzumessung strafmildernd berücksichtigt werden kann. Dies gilt insbesondere für die Fälle, in denen die Kammer das Vorliegen eines Mordmerkmals ablehnt und den Täter wegen Totschlags i.S.v. \$212 StGB verurteilt. Der BGH hat die strafmildernde Berücksichtigung von durch heimatliche Wertvorstellungen geprägten Beweggründen bei ausländisch stämmigen Tätern mehrfach für zulässig erklärt (vgl. u.a. BGH NStZ 1996, 80; BGH NStZ-RR 1997, 1; BGH StV 2002, 20 f.; BGH NStZ-RR 2007, 138 f.). Gemäß diesen Entscheidungen ist eine strafmindernde Berücksichtigung der kulturellen Prägung des Täters insbesondere dann denkbar, wenn der Täter seinen heimatlichen Wertvorstellungen noch sehr stark verhaftet war, weil er noch nicht lange in Deutschland gelebt hat (vgl. BGH StV 1994, 430 f.; BGH NStZ-RR 1997, 1). Indes akzeptierte der BGH die Strafmilderung teilweise auch, wenn der Täter trotz längeren Aufenthalts in Deutschland nicht in der Lage war, sich von seiner kulturellen Prägung zu lösen oder wenn er sich zwischen seinem heimatlichen und dem deutschen Kulturkreis »zerrissen « gefühlt hat (vgl. u.a. BGH NStZ 1982, 115 f. und BGH NStZ-RR 1997, 1). Diese Rechtsprechung steht somit im Einklang mit der oben skizzierten aktuellen Argumentation der Strafsenate hinsichtlich der Einordnung des Ehrmotivs als niedriger Beweggrund.

\section{Fragestellung und Ziele der Untersuchung}

Die Studie beschäftigte sich mit der Frage, wie die großen Strafkammern der deutschen Landgerichte das Phänomen der Ehrenmorde bewerten. Verurteilen sie die Täter überwiegend zu lebenslangen Freiheitsstrafen wegen Mordes aus niedrigen Beweggründen i.S.v. \211 StGB entsprechend der aktuellen Rechtsprechung des BGH? Oder nutzen sie häufig den vom BGH eingeräumten Ermessensspielraum, um die lebenslange Haftstrafe zu umgehen und entscheiden daher auf eine Bestrafung wegen Totschlags i.S.v. $\$ 212$ StGB? Führt der 
unscharfe Übergang der zweiten objektiven zur aktuellen subjektiven Rechtsprechungsphase des BGH zu widersprüchlichen Urteilen der Landgerichte? Wird die Sozialisation des Täters bei einer Verurteilung zu einer zeitigen Freiheitsstrafe als Strafmilderungsgrund gewertet? Zeichnet sich bei den Ehrenmorden eine Tendenz zu höheren Strafen ab - sei es wegen der Verfestigung der aktuellen, eher restriktiven Rechtsprechung durch den BGH oder wegen eines möglichen allgemeinen punitiven Trends der deutschen Rechtsprechung? Außerdem sollte die Frage beantwortet werden, ob die Schwurkammern die Taten deutschlandweit einheitlich beurteilen oder ob Ehrenmorde in Städten, in denen sie häufiger verübt werden, anders sanktioniert werden als in Regionen, in denen diese Taten seltener begangen werden. Die Studie zielte mit diesen Fragen darauf ab, ein umfassendes und repräsentatives Bild der justiziellen Praxis im Umgang mit dem Phänomen der Ehrenmorde in Deutschland zu zeichnen.

Zudem wurde die Frage untersucht, ob und inwieweit sich das Strafmaß in Ehrenmordfällen von den in anderen, ähnlichen Fällen von Tötungsdelinquenz verhängten Strafen unterscheidet. Insbesondere die auch von deutschen Männern verübten (Ex-)Intimpartnertötungen weisen hinsichtlich Motivlage und Täter-Opfer-Konstellation Parallelen zu den Ehrenmorden auf. Insofern erscheint fraglich, ob diese beiden Tötungsformen auch ähnlich sanktioniert werden oder ob die deutschen Strafkammern Ehrenmörder milder oder strenger bestrafen als (deutsche) Männer, die ihre früheren oder aktuellen Intimpartnerinnen getötet haben. Durch den Vergleich der Strafzumessung in den beiden Samples sollte zudem ein wichtiger Beitrag zur empirischen Strafzumessungsforschung geleistet werden. Eine Gegenüberstellung der Strafzumessungspraxis in zwei verschiedenen Stichproben von Tötungsdelikten wurde in Deutschland in dieser Form zuvor noch nicht durchgeführt.

\section{Methodisches Vorgehen und Zusammensetzung der Stichproben}

Die hier untersuchten Ehrenmordfälle wurden im Rahmen des bereits erwähnten Ehrenmordprojekts von Oberwittler und Kasselt erhoben (Oberwittler E Kasselt 2011). Das Projekt zielte auf eine Vollerhebung aller polizeilich registrierten versuchten und vollendeten Fälle von Ehrenmord ab, die im Zeitraum zwischen dem 01.01.1996 und dem 31.12.2005 in Deutschland verübt wurden. Um dieses Ziel zu erreichen, wurden zwei verschiedene Suchstrategien verfolgt: Zum einen wurde in polizeilichen Datenbanken nach potenziellen Fällen recherchiert, und zum anderen wurde eine bundesweite Analyse von Medienarchiven durchgeführt, um weitere mögliche Ehrenmordfälle zu identifizieren. Die Landeskriminalämter meldeten uns insgesamt 77 potenzielle Ehrenmordfälle. Im Volltextarchiv der Nachrichtenagentur dpa wurden in einem aufwändigen Suchverfahren aus ca. 92.500 Meldungen 40 zusätzliche mögliche Fälle von Ehrenmord selektiert. Von fünf weiteren Fällen, die in die Bruttostichprobe einbezogen wurden, erlangten wir durch Recherchen in Archiven von Regionalzeitungen sowie in Fallsammlungen im Internet Kenntnis. Zudem wurden wir durch Hinweise von Kollegen und anderen Personen auf drei weitere mögliche Ehrenmorde aufmerksam.

Insgesamt ergab die Suche in unterschiedlichen Informationsquellen somit eine Stichprobe von 125 potenziellen Fällen. Diese Bruttostichprobe reduzierte sich schrittweise auf die Nettostichprobe von 78 Fällen. 12 der 125 angeforderten Fälle (9,6 \%) wurden von den Staatsanwaltschaften nicht aufgefunden. 14 Akten $(11,2 \%)$ wurden uns nicht zugesandt, weil sie anderweitig versendet waren oder weil das Verfahren noch nicht abgeschlossen war. Von den 99 Fällen, die zur Einsicht vorlagen, wurden 21 (21,2\%) als falsch-positiv aussortiert, weil sie nicht den Kriterien der im Ehrenmordprojekt und in dieser Studie verwendeten Arbeitsdefinition entsprachen.

In die im Rahmen der hier vorgestellten Studie durchgeführten Urteilsauswertungen wurden nicht alle 78 Ehrenmordfälle einbezogen, sondern nur die 60 Fälle $(76,9 \%)$, in denen es 
zur Verurteilung mindestens eines Täters vor einem deutschen Gericht kam. Für die verschiedenen Analysen wurden außerdem weitere der 86 in Deutschland verurteilten Täter herausgefiltert, insbesondere die nach Jugendstrafrecht abgeurteilten Angeklagten sowie Täter, die nur wegen eines Körperverletzungsdelikts oder als Gehilfen i.S.v. \27 StGB verurteilt wurden. Für den Großteil der bivariaten und multivariaten Analysen wurde ein Subsample von 51 (Haupt-)Tätern/Fällen verwendet, dies entspricht 65,4 \% der 78 Fälle aus der Nettostichprobe.

Die Datensätze der hier vergleichend untersuchten Partnertötungsstichprobe wurden im Rahmen eines Parallelprojekts am Max-Planck-Institut für ausländisches und internationales Strafrecht Freiburg zum Thema »Familialer Homizid-Suizid « erhoben. Neben den Tötungsdelikten mit anschließendem Suizid wurde zu Vergleichszwecken auch eine Stichprobe von Partnertötungsfällen ohne Anschlusssuizid erhoben, welche für die vorliegende Untersuchung ausgewertet wurde.

Im Rahmen der Homizid-Suizid-Studie wurden vier Bundesländer ausgewählt, aus denen Partnertötungsfälle angefordert wurden: Mit Hamburg wurde ein Stadtstaat in die Stichprobe einbezogen, Sachsen wurde als ostdeutsches, Niedersachsen als norddeutsches und Baden-Württemberg als süddeutsches Flächenbundesland ausgewählt. Um die Menge der Fälle weiter einzugrenzen, wurden bei den Landeskriminalämtern dieser Bundesländer die Aktenzeichen aller Fälle vollendeter (Ex-)Intimpartnertötungen mit männlichem Täter ohne anschließenden Suizid(-versuch) aus den Jahren 1998, 2000, 2002 und 2004 erbeten. Auf diesem Weg erhielten wir Kenntnis von 186 potenziellen Partnertötungsfällen, welche dann bei den zuständigen Staatsanwaltschaften angefordert wurden. 91 der 186 Tötungsdelikte (48,9 \%) wurden in Baden-Württemberg begangen, 44 Fälle $(23,7 \%)$ wurden von niedersächsischen Staatsanwaltschaften angefordert, 22 Fälle (11,8 \%) fielen unter die Zuständigkeit der Hamburger Staatsanwaltschaft und die restlichen 29 Fälle (15,6 \%) wurden von den sächsischen Behörden erbeten.

110 der 186 Fälle (59,1\%) der Bruttostichprobe bildeten letztlich die Nettostichprobe. 49 Fälle $(26,3 \%)$ wurden uns von den Staatsanwaltschaften nicht zugeschickt, weil die Akten nicht auffindbar oder wegen anderweitiger Versendung nicht verfügbar waren. Von den verbleibenden 137 Fällen wurden 27 (19,7\%) als falsch-positiv herausgefiltert. Voraussetzung für die Einbeziehung eines Falls in die Stichprobe war, dass es sich um einen männlichen Täter handelte, der seine (Ex-)Intimpartnerin getötet hat. Versuchte Taten wurden von vornherein nicht von den Behörden angefordert oder herausgefiltert, wenn sie dennoch in die Bruttostichprobe gelangt waren. Die Nettostichprobe verteilt sich wie folgt auf die vier Bundesländer: 57 der 110 Taten (51,8\%) wurden in Baden-Württemberg begangen, $12(10,9 \%)$ in Hamburg, 13 Fälle $(11,8 \%)$ stammen aus Sachsen und $28(25,5 \%)$ aus Niedersachsen.

Für die Urteilsauswertungen waren auch hier nur die Fälle relevant, in denen der Täter vor einem deutschen Gericht verurteilt wurde. Dies betrifft 95 der 110 Fälle (86,4 \%). Zudem wurden für einen Großteil der quantitativen Analysen die Täter aussortiert, die nur wegen eines Körperverletzungsdelikts verurteilt wurden. Das Hauptsample für die bivariaten und multivariaten Analysen der Vergleichsstichprobe umfasst somit 91 Fälle/Täter (82,7 \% der 110 Täter).

Die Daten wurden mittels der Methode der Aktenanalyse erhoben. Das Strafaktenmaterial enthält die von Polizei, Staatsanwaltschaft und Gericht zusammengetragenen Informationen zu den Fällen, inklusive sämtlicher Zeugenaussagen sowie psychiatrischen und sonstigen Sachverständigengutachten. Damit konnte aus den Akten ein umfassender Eindruck hinsichtlich der Fälle gewonnen werden, und bei deren Bewertung konnten die verschiedenen Sichtweisen der beteiligten Akteure einbezogen werden. Allerdings handelt es sich bei 
dem Aktenmaterial naturgemäß um von den staatlichen Organen vorselektierte und prozessproduzierte Sekundärdaten, was die Gefahr einer gewissen Verzerrung birgt (vgl. Dölling 1984, 269). Dennoch erschien die Aktenanalyse für die hier untersuchte Fragestellung besser geeignet als die möglichen Alternativen (qualitative Interviews, Gruppendiskussionen, Prozessbeobachtung).

Für das Homizid-Suizid-Projekt wurde ein umfangreiches Erhebungsinstrument mit etwa 430 Variablen entwickelt, mit denen größtenteils in standardisierter, teilweise aber auch in nicht-standardisierter Form alle für die Fragestellung relevanten Informationen erfasst wurden. Die erhobenen Variablen erfassten folgende inhaltliche Dimensionen: Tathergang, familiäres Umfeld von Täter und Opfer sowie Opferverhalten im Kontext der Tat, soziodemografische Konstellationen von Täter und Opfer, Tatvorgeschichte und Motivlage, Persönlichkeit und Vorbelastungen des Täters, Nachtatverhalten des Täters, justizieller Umgang mit dem Fall. Das Erhebungsinstrument für das Ehrenmordprojekt baut auf dem Erhebungsbogen des Homizid-Suizid-Projektes auf. Die meisten Variablen wurden übernommen, um eine Vergleichbarkeit der Daten zu gewährleisten. Der Codebogen wurde für das Ehrenmordprojekt aber um zusätzliche für die Fragestellung des Ehrenmordprojektes relevante Variablen erweitert, insbesondere zu den Bereichen polizeilicher und justizieller Umgang mit der Tat, Vorgeschichte der Tat, Tatmotivation sowie zur Rolle möglicher Tatzeugen. Insgesamt wurden für das Ehrenmordprojekt etwa 570 Variablen erhoben. Da im Rahmen des Homizid-Suizid-Projektes nur wenige Informationen zur juristischen Bewertung der Fälle erfasst wurden, erfolgte für die hier vorgestellte Studie eine Nacherhebung der fehlenden Daten aus den Urteilskopien anhand des für das Ehrenmordprojekt entwickelten Erhebungsinstruments. Zudem wurden für beide Stichproben die Strafzumessungserwägungen der Kammern nacherhoben. Der entsprechende Codebogen umfasst 40 Variablen.

\section{Empirische Befunde}

Nachfolgend werden ausgewählte Ergebnisse der empirischen Studie vorgestellt. Zunächst wird dabei auf einige zentrale Befunde der Auswertung der Ehrenmordurteile eingegangen. Anschließend werden wesentliche Ergebnisse des Strafzumessungsvergleichs zwischen den Ehrenmord- und den Partnertötungsurteilen skizziert.

\subsection{Die Sanktionierung von Ehrenmorden durch die deutschen Schwurgerichts- kammern}

86 der 122 Täter/Tatverdächtigen (70,5\%) aus der Ehrenmordstichprobe wurden vor einem deutschen Gericht verurteilt. Acht Täter (6,6\%) wurden in dubio pro reo freigesprochen, die Täterschaft konnte ihnen nicht zweifelsfrei nachgewiesen werden. In weiteren acht Fällen kam es aus unterschiedlichen Gründen nicht zu einer Verurteilung vor einem deutschen Gericht. Zwei Täter waren zum Tatzeitpunkt erst 13 Jahre alt und damit gemäß $\$ 19$ StGB schuldunfähig beziehungsweise strafunmündig. Vier Tatverdächtige flüchteten nach der Tat ins Ausland. Sie wurden von den Heimatländern nie an die deutschen Behörden ausgeliefert. Ein Täter wurde für schuldunfähig i.S.v. $\$ 20$ StGB befunden. Gegen eine Tatverdächtige wurde wegen Beweismangels kein Ermittlungsverfahren eingeleitet, obwohl die ermittelnden Beamten aufgrund ihres Verhaltens sowie von Zeugenaussagen über Äußerungen der Frau davon überzeugt waren, dass diese als Gehilfin oder gar Anstifterin an der Tat beteiligt war. Bei 15 Tätern (12,3\%) wurde das Verfahren eingestellt. In einigen dieser Fälle hatte der Täter sich in der Untersuchungshaft das Leben genommen oder war ins Ausland geflüchtet, in anderen Fällen reichte die Beweislage gegen die Verdächtigen nicht für eine 
Anklage aus. Bei den restlichen fünf Tätern (4,1\%) blieb der Ausgang des Verfahrens uns unbekannt, weil die Akten einiger Täter vom Hauptverfahren abgetrennt wurden.

13 der 86 vor deutschen Gerichten verurteilten Täter der Ehrenmordstichprobe (15,1\%) wurden wegen eines Körperverletzungsdelikts bestraft, da ihnen der Tötungsvorsatz nicht zweifelsfrei nachgewiesen werden konnte. Die anderen 73 Täter (84,9\%) wurden wegen versuchten oder vollendeten Totschlags oder Mordes verurteilt. Gegen 32 Täter (37,2 \% der 86 Verurteilten) wurde eine Verurteilung wegen Mordes i.S.v. \$211 StGB ausgesprochen, gegen 21 dieser 32 Angeklagten (65,6\%) wegen Mordes aus niedrigen Beweggründen. Das Motiv der Ehre wurde allerdings nur bei 14 dieser 21 Täter (66,7 \%) als niedriger Beweggrund gewertet, bei dem restlichen Drittel wurden Motive wie Hass, Eifersucht oder Besitzdenken als besonders verwerflich erachtet. Das Ehrmotiv wurde somit nur in 19,2 \% der 73 Verurteilungen wegen eines versuchten oder vollendeten Tötungsdelikts als niedriger Beweggrund und damit als Mordmerkmal eingestuft. Dies ist einerseits darauf zurückzuführen, dass die Ehre lediglich in 50 der 73 Verurteilungen (68,5\%) wegen eines vorsätzlichen Tötungsdelikts von der Kammer zumindest als Teil eines Motivbündels oder als Hauptmotiv bewertet wurde. Zudem prüften die Kammern in 17 dieser 50 Verurteilungen (34,0 \%) zwar, ob das Ehrmotiv als niedriger Beweggrund zu werten ist, entschieden sich letztlich aber dagegen. In 19 der 50 Fälle (38,0 \%), in denen die Kammer die Ehre zumindest als Teilmotiv einschätzte, wurde die Frage, ob es sich beim Ehrmotiv um einen niedrigen Beweggrund handelt, im Urteil überhaupt nicht angesprochen.

Tabelle 1 zeigt, differenziert nach Ehrenmordtypus, bei wie vielen der 50 wegen eines Tötungsdelikts verurteilten Tätern das Motiv der Wiederherstellung der Ehre als niedriger Beweggrund und damit als Mordmerkmal im Sinne des $\$ 211$ StGB eingestuft wurde. Bei den Ehrenmorden im engeren Sinne war der Anteil von Urteilen, in denen der niedere Beweggrund der Ehre bejaht wurde, mit 52,6 \% erwartungsgemäß am höchsten (zehn Urteile), bei den Grenzfällen zur Partnertötung war er mit einem Fall (oder 8,3 \% der Urteile zu Partnertötungsgrenzfälle) am niedrigsten. Auch bei den Grenzfällen zur Blutrache sowie den sonstigen Fällen war der Anteil der Urteile, in denen die Ehre als niederer Beweggrund i.S.v. $\int 211$ Abs. 2 StGB gewertet wurde, sehr gering: Er betrug 18,2 \% (zwei Urteile) sowie $12,5 \%$ (ein Urteil).

\section{Tabelle $1 \quad$ Richterliche Bewertung des Ehrmotivs als niedriger Beweggrund nach Ebrenmordtypus}

\begin{tabular}{|c|c|c|c|c|c|}
\hline \multirow{2}{*}{$\begin{array}{l}\text { Ehrmotiv als } \\
\text { niedriger } \\
\text { Beweggrund }\end{array}$} & \multicolumn{4}{|c|}{ Ehrenmordtypus } & \multirow[b]{2}{*}{ Gesamt } \\
\hline & $\begin{array}{c}\text { Ehrenmord } \\
\text { i.e.S. }\end{array}$ & $\begin{array}{l}\text { Grenzfall zur } \\
\text { Partnertötung }\end{array}$ & $\begin{array}{l}\text { Grenzfall zur } \\
\text { Blutrache }\end{array}$ & sonstige & \\
\hline \multirow{2}{*}{ nicht geprüft } & 5 & 7 & 6 & 1 & 19 \\
\hline & $26,3 \%$ & $58,4 \%$ & $54,5 \%$ & $12,5 \%$ & $38,0 \%$ \\
\hline \multirow{2}{*}{$\begin{array}{l}\text { geprüft, aber } \\
\text { abgelehnt }\end{array}$} & 4 & 4 & 3 & 6 & 17 \\
\hline & $21,1 \%$ & $33,3 \%$ & $27,3 \%$ & $75,0 \%$ & $34,0 \%$ \\
\hline \multirow{2}{*}{ angenommen } & 10 & 1 & 2 & 1 & 14 \\
\hline & $52,6 \%$ & $8,3 \%$ & $18,2 \%$ & $12,5 \%$ & $28,0 \%$ \\
\hline \multirow{2}{*}{ Gesamt } & 19 & 12 & 11 & 8 & 50 \\
\hline & $100,0 \%$ & $100,0 \%$ & $100,0 \%$ & $100,0 \%$ & $100,0 \%$ \\
\hline
\end{tabular}

Nur Verurteilungen wegen Tötungsdelikten, bei denen die Ehre als Motiv gesehen wurde, $\mathrm{N}=50$ Täter 
Vor dem Hintergrund der aktuellen Rechtsprechung des BGH, wonach das Ehrmotiv auf objektiver Ebene grundsätzlich als niedriger Beweggrund zu bewerten ist und nur auf subjektiver Ebene ausnahmsweise die besondere Verwerflichkeit der Tat ausgeschlossen sein kann, erscheint insbesondere die hohe Zahl von Urteilen, in denen diese Problematik nicht angesprochen wurde, überraschend - vor allem wegen des Risikos einer erfolgreichen Revision des Urteils. Vorstellbar ist, dass manche Kammern auf die Prüfung der schwierig zu beantwortenden Frage des Vorliegens niedriger Beweggründe verzichtet haben, weil bereits ein anderes Mordmerkmal für einschlägig befunden wurde und es daher für eine Verurteilung wegen Mordes nicht mehr auf die Frage des Vorliegens eines niederen Beweggrundes ankam. Diese Erklärung kommt bei 7 der 19 Täter (36,9 \%) in Betracht, die nach Auffassung der Kammer mindestens ein anderes Mordmerkmal verwirklicht hatten. In weiteren sieben Fällen, in denen nicht geprüft wurde, ob es sich bei der Ehre um einen niedrigen Beweggrund handelt, würdigte die Kammer die heimatlichen Wertvorstellungen des Täters als Strafmilderungsgrund. Dies zeigt, dass die Richter die Beweggründe eben nicht als besonders verwerflich bewerteten und wohl daher auf die Prüfung verzichteten. Warum in den anderen fünf Fällen (26,3\%) auf die Prüfung des Mordmerkmals »niedrige Beweggründe « verzichtet wurde, kann nur spekuliert werden: Möglicherweise wollten die Strafkammern diese schwierige Frage nicht aufwerfen, weil sie befürchteten, dass ihre Erwägungen dazu einer Revision nicht standhalten würden. Es ist evident, dass die Frage, wie stark der Täter noch in seinen heimatlichen Wertvorstellungen verankert ist, für deutsche Richter nicht einfach zu beurteilen ist. Allerdings holten sich die Juristen selten Rat bei Spezialisten: Bei nur 11 der 73 Verurteilungen $(15,1 \%)$ wegen eines vorsätzlichen Tötungsdelikts wurde ein ethnologisches oder ethnopsychologisches Gutachten eingeholt. In den anderen Fällen fühlten sich die Richter scheinbar selbst in der Lage dazu, den kulturellen Hintergrund der Tat richtig einzuordnen.

Aufschlussreich ist auch der Blick auf die 17 Fälle, in denen die Kammern Erwägungen zum Vorliegen eines niedrigen Beweggrundes anstellten, aber sich letztlich dagegen entschieden. Bei sieben der 17 Täter (41,2\%) wurde die Verwerflichkeit des Ehrmotivs schon auf objektiver Ebene abgelehnt. Dies spricht dafür, dass ein Teil der Kammern sich - vermutlich wegen des BGH-Urteils aus dem Jahr 1997 - noch an der früheren Rechtsprechung des BGH orientiert hat. Bei den anderen zehn Tätern $(58,8 \%)$ wurde die innere Tatseite des niederen Beweggrundes abgelehnt, meist mit der Begründung, dass der Täter noch stark in seinen heimatlichen Wertvorstellungen verhaftet gewesen sei. Die Anzahl dieser Fälle sowie der Fakt, dass so auch bei Tätern argumentiert wurde, die zum Tatzeitpunkt schon jahrzehntelang in Deutschland lebten, deuten darauf hin, dass die Gerichte die Ausnahmeregelung des BGH eher weit auslegen - möglicherweise, um die Verhängung einer lebenslangen Freiheitsstrafe zu vermeiden.

Der kulturelle Hintergrund der Tat wurde bei 13 Tätern (26,0 \% der Täter, bei denen die Ehre zumindest als Teilmotiv erachtet wurde) im Rahmen der Strafzumessung strafmildernd berücksichtigt. Strafschärfend wurde er hingegen nur bei zwei Tätern (2,7 \% der 73 wegen eines Tötungsdelikts Verurteilten) herangezogen.

Abbildung 1 veranschaulicht die Höhe des Strafmaßes, welches gegen die wegen eines Tötungsdelikts Verurteilten verhängt wurde. Die Verurteilungen nach Jugendstrafrecht wurden hier nicht einbezogen, da die Jugendstrafe aufgrund ihrer abweichenden Strafrahmen (vgl. \$18 Abs. 1 JGG) nicht mit der Freiheitsstrafe nach allgemeinem Strafrecht vergleichbar ist. Die Darstellung bezieht sich somit auf ein Teilsample von 63 Tätern, die nach allgemeinem Strafrecht wegen eines Tötungsdelikts verurteilt wurden. Wie bei vorsätzlichen Tötungsdelikten zu erwarten, handelt es sich um eine linksschiefe Verteilung mit dem Peak bei den lebenslangen Freiheitsstrafen. 31,7 \% der Täter wurden zu einer lebenslangen Frei- 
heitsstrafe verurteilt, bei vier Tätern (6,3\%) wurde zusätzlich die besondere Schwere der Schuld festgestellt. Bei diesen Tätern ist gemäß $\$ 57$ a Abs. 1 Nr. 2 StGB eine Strafrestaussetzung nach 15 Jahren nicht möglich. Insgesamt wurden somit 24 Täter $(38,1 \%)$ der Ehrenmordstichprobe zu einer lebenslangen Freiheitsstrafe verurteilt.

Abbildung 1 Verbängtes Strafmaß beiden Verurteilungen wegen eines Tötungsdelikts in der Ebrenmordstichprobe

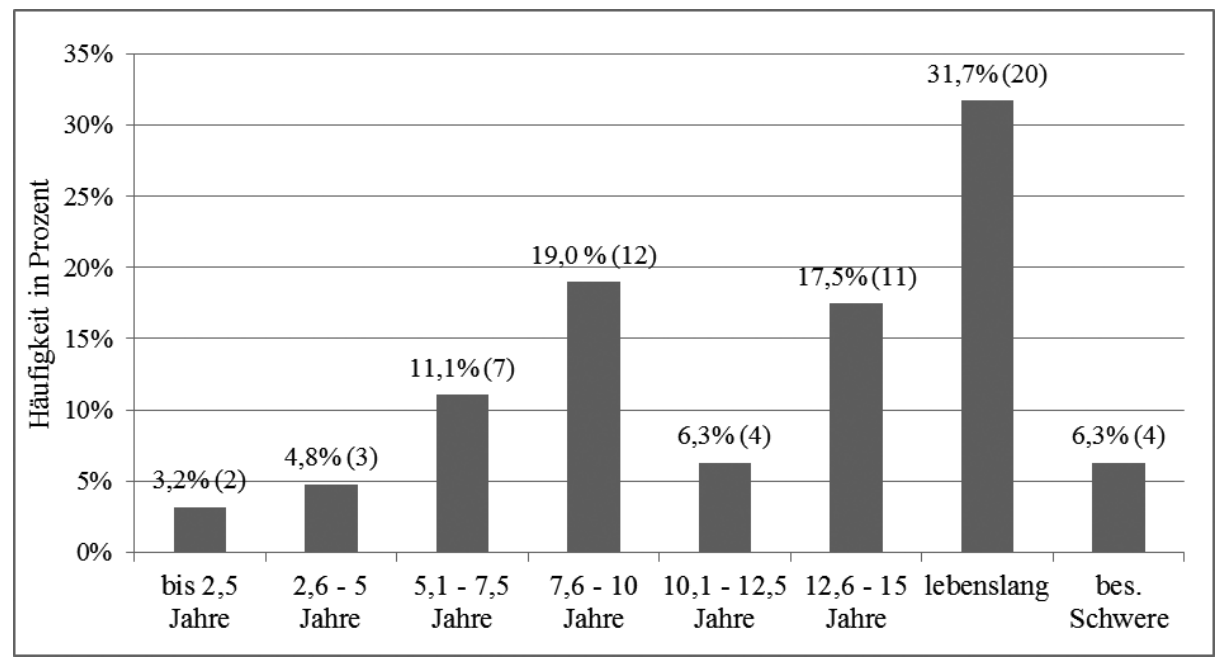

$\mathrm{N}=63$ Verurteilte, ohne Jugendstrafe (Angaben in Prozent, absolute Zahlen in Klammern)

Ein Schwerpunkt der hier vorgestellten Studie war die Überprüfung des Einflusses verschiedener Faktoren auf das in den Ehrenmordfällen verhängte Strafmaß mittels bi- und multivariater Analysen. In diesem Rahmen können nur wenige zentrale Befunde dieser Analysen skizziert werden, für Details sei auf die Ende 2014/Anfang 2015 zu erwartende Monographie »Ehre im Spiegel der Justiz« verwiesen.

Die für das multiple Regressionsmodell verwendete abhängige Variable »Strafmaß « wurde wie folgt skaliert: 1 = Freiheitsstrafe bis zu zehn Jahren«, $2=»$ Freiheitsstrafe zwischen 10 und 15 Jahren«, 3 = »lebenslange Freiheitsstrafe«. Da diese Variable ein ordinales Skalenniveau aufweist, wurde als Verfahren zur Berechnung der multivariaten Einflüsse die ordinale Regression gewählt. ${ }^{6}$ Die unabhängigen Variablen des Modells lauten »Tatvorbereitung « $(0=$ nein $/ 1=j \mathrm{j})$, »Anzahl der Todesopfer $«(0=»$ kein Todesopfer $\ll / 1=»$ ein Todesopfer/2 = »zwei oder mehr Todesopfer«), »verminderte Schuldfähigkeit« $(0=$ nein/1 = ja), »Ehrenmordtypus « $(1=»$ Ehrenmord im engeren Sinne $/ 2=»$ Partnertötungsgrenzfall $/ / 3=$ »Sonstiges «) sowie »Urteilsjahr« ( 0 = bis $2001 \ll / 1=»$ nach 2001 «). Letztere Variable wurde dichotomisiert, weil im Rahmen der bivariaten Analysen eine Zweiteilung hinsichtlich der verhängten Strafen deutlich geworden ist: Ab dem Jahr 2002 wurden mehr lebenslange Freiheitsstrafen verhängt und die Höhe der verhängten zeitigen Freiheitsstrafen stieg erkennbar

6 Vgl. zu diesem Verfahren ausführlich Kühnel 2010, 855 ff. Auch das allgemeine lineare Modell kam als Analyseverfahren in Betracht. Da hierfür allerdings im Gegensatz zur ordinalen logistischen Regression kein Pseudo-Bestimmtheitsmaß (vgl. dazu ausführlich u.a. Backhaus 2008, 263 ff.) berechnet werden kann, welches den erklärten Anteil der Variabilität des Gesamtmodells angibt, fiel die Wahl auf die logistische Regression. 
Tabelle 2

Schätzwerte des ordinalen Regressionsmodells für das verbängte Strafmaß in der Ehrenmordstichprobe

\begin{tabular}{|c|c|c|c|c|c|c|}
\hline & \multicolumn{5}{|c|}{ Parameterschätzer } \\
\hline & & B (Logit) & $\begin{array}{l}\text { Std.- } \\
\text { Fehler } \\
\end{array}$ & Wald & Sig. & $\begin{array}{l}\text { Odds } \\
\text { Ratio }\end{array}$ \\
\hline \multirow{2}{*}{$\begin{array}{l}\text { Schwellen (Re- } \\
\text { ferenz: lebens- } \\
\text { lange Freibeits- } \\
\text { strafe) }\end{array}$} & $\begin{array}{l}\text { Strafmaß = bis 10 Jahre } \\
\text { Freiheitsstrafe }\end{array}$ & 1,595 & 0,973 & 2,687 & 0,101 & \\
\hline & $\begin{array}{l}\text { Strafmaß }=10 \text { bis } 15 \text { Jahre } \\
\text { Freiheitsstrafe }\end{array}$ & 3,673 & 1,107 & 11,016 & 0,001 & \\
\hline \multirow{7}{*}{ Prädiktoren } & $\begin{array}{l}\text { Urteilsjahr nach } 2001 \\
\text { (Ref.= bis 2001) }\end{array}$ & 3,392 & 0,948 & 12,800 & 0,000 & 29,73 \\
\hline & $\begin{array}{l}\begin{array}{l}\text { Tatvorbereitung }(\text { Ref. }= \\
\text { keine) }\end{array} \\
\end{array}$ & 1,385 & 0,732 & 3,585 & 0,058 & 3,99 \\
\hline & $\begin{array}{l}\text { verminderte Schuldfähig- } \\
\text { keit (Ref.= nein) } \\
\end{array}$ & $-2,541$ & 1,122 & 5,131 & 0,024 & 0,08 \\
\hline & $\begin{array}{l}\text { Anzahl der Todesopfer }= \\
\text { O(Ref. }=1)\end{array}$ & $-1,555$ & 1,147 & 1,838 & 0,175 & 0,21 \\
\hline & $\begin{array}{l}\text { Anzahl der Todesopfer }= \\
2+(\text { Ref. }=1)\end{array}$ & 2,638 & 1,609 & 2,686 & 0,101 & 13,99 \\
\hline & $\begin{array}{l}\text { Typ »Ehrenmord i.e.S.» } \\
\text { (Ref. = Grenzfall Part- } \\
\text { nertötung) } \\
\end{array}$ & 3,087 & 1,267 & 5,939 & 0,015 & 21,91 \\
\hline & $\begin{array}{l}\text { Typ »Sonstige« (Ref. }= \\
\text { Grenzfall Partnertötung) }\end{array}$ & $-0,107$ & 0,799 & 0,018 & 0,893 & 0,90 \\
\hline
\end{tabular}

Ordinales Regressionsmodell, $\mathrm{N}=50,-2 \mathrm{LL}=44,01, \mathrm{X}^{2}=42,2$ (7 df), $\mathrm{p}<0,001$, Pseudo- $\mathrm{R}^{2}$ (Nagelkerke) $=0,654,54,2 \%$ der Zellen mit Häufigkeit $=0$.

an. Um diesen Trend zu härteren Strafen im multivariaten Modell zu überprüfen, wurde daher die dichotome Variable »Urteilsjahr« gebildet. Auch bei den anderen unabhängigen Variablen hatte sich auf bivariater Ebene ein starker Zusammenhang mit der Höhe des Strafmaßes gezeigt, weshalb sie ins multivariate Modell übernommen wurden.

Tabelle 2 zeigt die Schätzwerte des multiplen Modells. Die hohen Logits und Odds Ratio-Werte machen deutlich, dass das Urteilsjahr auch unter Kontrolle der anderen unabhängigen Variablen einen starken und höchst signifikanten positiven Einfluss auf das verhängte Strafmaß hat. In den Urteilen seit dem Jahr 2002 wurden deutlich mehr lebenslange Freiheitsstrafen sowie höhere zeitige Freiheitsstrafen verhängt als in den Urteilen, die aus der Zeit bis 2001 stammen.

Als Erklärung für diesen Befund könnte das BGH-Urteil vom 20.02.2002 in Betracht kommen, in welchem der 5. Strafsenat die Grundsatzentscheidung aus dem Jahr 1994 bestätigte, wonach das Ehrmotiv prinzipiell als niedriger Beweggrund i.S.v. $\$ 211 \mathrm{StGB}$ zu werten ist und bei einer Tötung aus Gründen der Ehre nur ausnahmsweise eine Verurteilung wegen Totschlags in Betracht kommt (vgl. BGH NStZ 2002, 369 f.). Möglicherweise hatte erst die Wiederholung dieser Auffassung einen entscheidenden Effekt auf die Rechtsprechung der Landgerichte, weil die Entscheidung aus dem Jahr 1994 von den Strafkammern wegen des der vorherigen Rechtsprechungslinie zuzuordnenden Urteils aus dem Jahr 1997 (vgl. BGH StV 1997, 566) noch nicht als Rechtsprechungswandel wahrgenommen worden war.

Zudem betonte der BGH in der Entscheidung vom Februar 2002 den Ausnahmecharakter einer Verurteilung wegen Totschlags bei einer Tötung zur Wiederherstellung der Ehre stär- 
ker als im Urteil aus dem Jahr 1994. ${ }^{7}$ Es ist denkbar, dass die Formulierung des ersten Urteils von den Richtern der Landgerichte hinsichtlich des Regel-Ausnahme-Verhältnisses als weniger strikt wahrgenommen wurde als die eindeutigere Formulierung des Urteils aus dem Jahr 2002.

Der Anstieg der Strafhöhen bei den Ehrenmorden könnte aber auch auf einen generellen punitiven Trend bei Tötungsdelikten hinweisen. Dies legen Strengs Ergebnisse seiner Untersuchung der Sanktionspraxis bei Tötungsdelikten zwischen 1988 und 2007 nahe (vgl. Streng 2011, 57). Wenn die Straferhöhung bei den Ehrenmorden tatsächlich auf eine solche generelle punitive Tendenz zurückzuführen sein sollte, müsste sich auch bei der Vergleichsstichprobe von Partnertötungsfällen ein entsprechender Anstieg der Strafhöhen feststellen lassen. Die Ergebnisse der entsprechenden Analysen werden unter 6.2 dargestellt.

Erwartungsgemäß hat auch die verminderte Schuldfähigkeit des Täters im multivariaten Modell weiterhin einen deutlichen und signifikanten negativen Effekt auf das Strafmaß. Der Einfluss der Variablen »Anzahl der Todesopfer «, »Tatvorbereitung « und »Ehrenmordtypus « bleibt unter Kontrolle der anderen Variablen ebenfalls erhalten, der Zusammenhang fällt allerdings schwächer aus als auf der bivariaten Ebene und die Ergebnisse sind zum Teil nur schwach signifikant. Innerhalb der Variable »Ehrenmordtypus « zeigen sich klare Unterschiede: Die Ehrenmorde im engeren Sinne werden im Vergleich zur Referenzgruppe der Partnertötungsgrenzfälle signifikant härter bestraft, während die Täter der Gruppe der sonstigen Fälle, also der Blutrachegrenzfälle und Mischfälle, milder bestraft werden als die Angeklagten in den Partnertötungsgrenzfällen, wobei dieser Effekt nicht mehr signifikant ist. Die bivariat gefundenen Ergebnisse bleiben somit auch unter Kontrolle der anderen unabhängigen Variablen weitestgehend erhalten. Die Varianz der Variable »Strafmaß « wird durch das Regressionsmodell gut abgebildet: Aus dem Wert des Pseudo-Bestimmtheitsmaßes geht hervor, dass 65,4 \% der Varianz durch das Modell erklärt werden. ${ }^{8}$

Für regionale Unterschiede in der Sanktionierung der Ehrenmorde wurden keine Belege gefunden. Zwar wurden in Hessen signifikant mehr lebenslange Freiheitsstrafen als in den anderen Bundesländern verhängt, dies war aber auf andere Faktoren - die Opferzahl, die Frage der Schuldfähigkeit des Täters, den Ehrenmordtypus und das Tatstadium - zurückzuführen, wie im Rahmen der multivariaten Analysen festgestellt wurde.

Ein weiterer Befund der Untersuchung ist der Einfluss der kulturellen Assimilation des Täters auf das Strafmaß in den Ehrenmordfällen. Hinsichtlich der Entscheidung der Kammern über die Verhängung einer lebenslangen Freiheitsstrafe zeigte sich ein positiver Effekt der Assimilation des Täters: Gegen Täter, die sich stärker an die deutschen Werte und Normen angepasst hatten und weniger intensiv mit den heimatlichen Traditionen verbunden waren, wurden häufiger lebenslange Freiheitsstrafen verhängt als gegen Täter, die (fast) aus-

7 Vgl. den 2. Leitsatz des Urteils von 2002: »Nur ausnahmsweise [...] kann anstatt einer Verurteilung wegen Mordes aus niedrigen Beweggründen lediglich eine Verurteilung wegen Totschlages in Betracht kommen«(BGH NStZ 2002, 369) und die Formulierung von 1994 im 2. Leitsatz: »Diese Bewertung führt allerdings dann nicht zu einer Verurteilung wegen Mordes aus niedrigen Beweggründen, wenn [...]« (BGH NStZ 1995, 79). Zusätzlich wurde 1994 auch noch der komplette 3. Leitsatz dieser Ausnahme gewidmet, wodurch der Schwerpunkt eher auf der Ausnahmeregelung als auf der Regel liegt, so dass diese Ausnahme möglicherweise von den Richtern der Schwurkammern weiter ausgelegt wurde (vgl. BGH NStZ 1995, 79).

8 Als Maßzahl wurde hier Nagelkerkes $\mathrm{R}^{2}$ gewählt, weil bei diesem im Gegensatz zu Cox und Snells $\mathrm{R}^{2}$ sowie $M c F$ addens $\mathrm{R}^{2}$ ein Maximalwert von 1 erreicht werden kann, was die Interpretation erleichtert (vgl. Backhaus 2008, 264). Angesichts der geringen Stichprobengröße und wegen der Schätzproblematik in nichtlinearen Regressionsmodellen sollte das Pseudo- $\mathrm{R}^{2}$ zurückhaltend interpretiert werden und ist nicht mit dem entsprechenden Maß einer OLS-Regression vergleichbar. 
schließlich nach heimatlichen Werten und Traditionen lebten, sich also der deutschen Kultur nicht geöffnet hatten. Dieses Ergebnis leuchtet im Hinblick auf die aktuelle Rechtsprechung des BGH ein, denn danach können Ehrenmörder ausnahmsweise statt wegen Mordes wegen Totschlags verurteilt werden, wenn sie sich nicht aus ihren heimatlichen Anschauungen lösen konnten. Bemerkenswert ist allerdings der Befund, dass sich eine höhere Assimilation des Täters negativ auf die Höhe der zeitigen Freiheitsstrafen auswirkt: Stärker integrierte Täter erhielten also niedrigere zeitige Freiheitsstrafen als weniger assimilierte Täter. Dies spricht dafür, dass die Gerichte in den Fällen, in denen sie sich wegen der starken Verwurzelung des Täters in den heimatlichen Wertvorstellungen gegen das Vorliegen eines niedrigen Beweggrundes und damit gegen die Verhängung einer lebenslangen Freiheitsstrafe entschieden haben, hohe Freiheitsstrafen verhängen, gewissermaßen als Ausgleich. Grund dafür könnte eine (generelle) Vermeidungstaktik der Gerichte hinsichtlich der Verhängung von lebenslangen Freiheitsstrafen sein. ${ }^{9}$

\subsection{Unterschiede und Parallelen in der Sanktionierung von Ehrenmorden und Partnertötungsfällen}

Der Anteil an lebenslangen Freiheitsstrafen lag in der Partnertötungsstichprobe mit 18,7 \% (17 von 91 Tätern, die wegen eines Tötungsdelikts verurteilt wurden) deutlich niedriger als in der Ehrenmordstichprobe. Dies ist zum einen mit dem etwas niedrigeren Anteil an Verurteilungen wegen Mordes zu erklären, zum anderen ist dieser Unterschied auf den deutlich höheren Anteil an für vermindert schuldfähig befundenen Tätern ( $\$ 21 \mathrm{StGB}$ ) in der Partnertötungsstichprobe zurückzuführen. Dieser lag mit 40,0\% in der gesamten Stichprobe etwa doppelt so hoch wie bei den Ehrenmorden (20,9 \%). Hinsichtlich der Verurteilungen wegen Mordes betrug der Anteil vermindert schuldfähiger Täter bei den Partnertötungen $26,7 \%$, in der Ehrenmordstichprobe hingegen nur 10,3 \%. Auch der Anteil an zeitigen Freiheitsstrafen zwischen 12,6 und 15 Jahren liegt in der Partnertötungsstichprobe mit 9,9\% auffällig niedriger als in der Ehrenmordstichprobe (17,5\%). Gleichzeitig wurden bei den Partnertötungen deutlich mehr Strafen zwischen 10 und 12,5 Jahren verhängt (18,7 \% vs. $6,3 \%$ ). Auch dieser Unterschied ist vermutlich vorrangig auf den deutlich höheren Anteil von vermindert schuldfähigen Tätern in der Partnertötungsstichprobe zurückzuführen: Bei den Verurteilungen wegen Totschlags lag er in der Partnertötungsstichprobe bei $45,9 \%$, bei den Ehrenmorden betrug dieser Anteil hingegen nur 23,5\%.

Ein Anstieg der Strafhöhen wurde in der Partnertötungsstichprobe nicht nachgewiesen. Sowohl hinsichtlich der Dauer der verhängten zeitigen Freiheitsstrafen als auch bezüglich der Anzahl der verhängten lebenslangen Freiheitsstrafen ist über den untersuchten Zehnjahreszeitraum kein signifikanter Trend feststellbar. Daher ist davon auszugehen, dass der bei den Ehrenmorden nachgewiesene Trend zu höheren Strafen nicht auf eine allgemeine punitive Tendenz bei Tötungsdelikten zurückzuführen ist. Vielmehr ist anzunehmen, dass das oben erwähnte BGH-Urteil vom Februar 2002 zu den deutlich härteren Sanktionen geführt hat.

Da die Teilgruppe der Partnertötungsgrenzfälle den Partnertötungsfällen aus der Vergleichsstichprobe stark ähnelt und sich die Taten vorrangig durch das Ehrmotiv und die damit verbundene kollektive Komponente unterscheiden, erschien ein Vergleich der in diesen beiden Fallgruppen verhängten Strafen im multivariaten Modell besonders interessant. Daher wurde ein entsprechendes übergreifendes Regressionsmodell erstellt. Als abhängige Va-

9 Vgl. zur These der Vermeidung von lebenslangen Freiheitsstrafen durch die Schwurkammern Sessar $1980,193 \mathrm{ff}$. 
riable für dieses Modell wurde eine vierstufige Version der Variablen »Strafmaß« gewählt: Die Kategorien 4 und 5 des Merkmals »Strafmaß « wurden aufgrund des geringen Anteils von Fällen, in denen die besondere Schuldschwere festgestellt wurde, zusammengelegt. Die Variable »Strafmaß vierstufig « ist somit wie folgt skaliert: 1 = »bis zu 5 Jahre Freiheitsstrafe«, $2=» 5$ bis 10 Jahre Freiheitsstrafe «, $3=» 10$ bis 15 Jahre Freiheitsstrafe « und $4=»$ lebenslange Freiheitsstrafe«. Aufgrund des ordinalen Skalenniveaus dieser abhängigen Variablen wurde erneut eine ordinale Regression berechnet.

Als Prädiktoren wurden die Merkmale »Tatvorbereitung «, »verminderte Schuldfähigkeit«, »Anzahl der Todesopfer « und »konfliktverschärfende Vorszene $(0=$ nein, $1=j a)$ ins Modell übernommen. Bei diesen Variablen hatte sich sowohl auf bivariater als auch auf multivariater Ebene ein deutlicher Effekt auf das Strafmaß in den beiden Stichproben gezeigt. Zudem wurde ein Interaktionseffekt für die Variablen »Urteilsjahr« $(0=»$ bis $2001 \ll / 1$ $=» a b 2002 \ll)$ und »Stichprobe $(0=\mathrm{EM} / 1=\mathrm{PT})$ berechnet, da in der Ehrenmordstichprobe ein starker Zusammenhang zwischen dem Urteilsjahr und dem Strafmaß gefunden wurde, während bei den Partnertötungen kein solcher Effekt nachgewiesen werden konnte. Die beiden Variablen und der Interaktionseffekt wurden ebenfalls ins multivariate Modell übernommen.

Tabelle 3 zeigt die Ergebnisse des Regressionsmodells. Bei allen unabhängigen Variablen zeigt sich ein mindestens schwach, teilweise aber auch hoch signifikanter Einfluss auf die Strafhöhen. Am schwächsten - und schwächer als in den multivariaten Modellen für beide Stichproben - fällt der positive Zusammenhang zwischen den Strafhöhen und der Frage der Tatvorbereitung aus. Das Vorliegen einer verminderten Schuldfähigkeit hat demgegenüber auch in diesem Modell einen starken und höchst signifikanten strafmindernden Effekt. Geringere Strafen werden außerdem in Fällen verhängt, denen ein konfliktverschärfendes Ereignis vorausgegangen ist, sowie bei Taten, bei denen kein Opfer gestorben ist. Wenn hingegen zwei oder gar mehr Opfer getötet wurden, erhielten die Täter erwartungsgemäß deutlich höhere Strafen. Das Modell erreicht mit einem Pseudo- $\mathrm{R}^{2}=49,2 \%$ eine hohe Erklärungskraft.

Aufschlussreich ist der Interaktionseffekt der Variablen »Stichprobe« und »Urteilsjahr«: Aus den Logits ergibt sich, dass der Einfluss der Variablen »Urteilsjahr« den straferhöhenden Effekt der Variablen »Stichprobe« umkehrt. ${ }^{10}$ Das heißt, in Urteilen aus der Zeit vor 2002 wurden in Ehrenmordfällen deutlich niedrigere und ab 2002 deutlich höhere Strafen verhängt als in den Fällen der vergleichenden Partnertötungsstichprobe.

Abbildung 2 illustriert die Auswirkungen dieses Interaktionseffektes auf das in beiden Stichproben verhängte Strafmaß anhand der Wahrscheinlichkeiten der Verhängung eines bestimmten Strafmaßes. Die Wahrscheinlichkeiten wurden aus den vorhergesagten Werten des ordinalen Regressionsmodells berechnet. Es wird deutlich, dass in den Partnertötungsfällen der Vergleichsstichprobe die Wahrscheinlichkeiten für die Verhängung der verschiedenen Strafmaßkategorien über den gesamten Urteilszeitraum konstant bleiben. Bei den der Ehrenmordstichprobe zuzurechnenden Partnertötungsgrenzfällen zeigen sich hingegen große Unterschiede zwischen den bis 2001 verhängten Strafhöhen und den ab 2002 ausgesprochenen Strafen. Die Wahrscheinlichkeit der Verhängung einer Strafe zwischen fünf und zehn Jahren sinkt ab 2002 auf $4 \%$, in den Jahren zuvor betrug sie noch $51 \%$. Auch die Wahrscheinlichkeit, dass die Kammer eine Strafe zwischen 10 und 15 Jahren verhängt, verringert

10 Unter Einschluss des Interaktionseffekts ergibt sich für die Wertekombination Stichprobe = Ehrenmord und Urteilsjahr $=\mathrm{ab} 2002$ ein Logit von $(1,626 * 0+3,318 * 1-3,209 * 0 * 1)=3,318$, für die Kombination Stichprobe $=$ Partnertötung und Urteilsjahr $=$ ab 2002 ergibt sich 1,626*1 + 3,318*1$3,209 * 1 * 1)=1,74$. 
Tabelle 3 Schätzwerte des ordinalen Regressionsmodells für »Strafmaß vierstufig»

\begin{tabular}{|c|c|c|c|c|c|c|}
\hline & \multicolumn{5}{|c|}{ Parameterschätzer } \\
\hline & & $\begin{array}{c}\mathrm{B} \\
\text { (Logit) } \\
\end{array}$ & $\begin{array}{l}\text { Std.- } \\
\text { Fehler } \\
\end{array}$ & Wald & Sig. & $\begin{array}{l}\text { Odds } \\
\text { Ratio }\end{array}$ \\
\hline \multirow{3}{*}{$\begin{array}{l}\text { Schwellen } \\
\text { (Ref.: lebens- } \\
\text { lange Freibeits- } \\
\text { strafe) }\end{array}$} & $\begin{array}{l}\text { Strafmaß = bis } 5 \text { Jahre } \\
\text { Freiheitsstrafe }\end{array}$ & $-3,577$ & 1,084 & 10,9 & 0,001 & \\
\hline & $\begin{array}{l}\text { Strafmaß = bis } 10 \text { Jahre } \\
\text { Freiheitsstrafe }\end{array}$ & 0,142 & 1,038 & 0,0 & 0,891 & \\
\hline & $\begin{array}{l}\text { Strafmaß }=10 \text { bis } 15 \text { Jahre } \\
\text { Freiheitsstrafe } \\
\end{array}$ & 1,967 & 1,049 & 3,5 & 0,061 & \\
\hline \multirow{8}{*}{ Prädiktoren } & $\begin{array}{l}\text { Tatvorbereitung (Ref.= } \\
\text { keine) }\end{array}$ & 0,904 & 0,423 & 4,6 & 0,033 & 2,469 \\
\hline & $\begin{array}{l}\text { verminderte Schuldfähig- } \\
\text { keit }(\text { Ref.= nein) }\end{array}$ & $-1,989$ & 0,473 & 17,7 & 0,000 & 0,137 \\
\hline & $\begin{array}{l}\text { konfliktverschärfende } \\
\text { Vorszene (Ref.= keine) }\end{array}$ & $-1,392$ & 0,566 & 6,1 & 0,014 & 0,249 \\
\hline & $\begin{array}{l}\text { Anzahl der Todesopfer }=0 \\
(\text { Ref. }=1)\end{array}$ & $-1,696$ & 0,955 & 3,2 & 0,076 & 0,183 \\
\hline & $\begin{array}{l}\text { Anzahl der Todesopfer }= \\
2+(\text { Ref. }=1)\end{array}$ & 2,376 & 0,914 & 6,8 & 0,009 & 10,762 \\
\hline & $\begin{array}{l}\text { Stichprobe Partnertötung } \\
\text { (Ref.: Ebrenmorde) }\end{array}$ & 1,626 & 0,903 & 3,2 & 0,072 & 5,083 \\
\hline & $\begin{array}{l}\text { Urteilsjahr ab } 2002 \text { (Ref.: } \\
\text { bis 2001) }\end{array}$ & 3,318 & 1,000 & 11,0 & 0,001 & 27,605 \\
\hline & $\begin{array}{l}\text { Interaktion Stichpro- } \\
\text { be*Urteilsjahr }\end{array}$ & $-3,209$ & 1,087 & 8,7 & 0,003 & 0,040 \\
\hline
\end{tabular}

Ordinales Regressionmodell, N = 114 Verurteilungen (91 Partnertötungen, 23 Grenzfälle zur Partnertötung), -2LL = 99,6 X $\mathrm{X}^{2}=68,1$ (8 df), $\mathrm{p}<0,001$, Pseudo-R ${ }^{2}$ (Nagelkerke) 0.492, 59,8\% der Zellen mit Häufigkeit $=0$.

sich bei den Urteilen aus der Zeit ab 2002 merklich (17\% vs. $34 \%$ bei älteren Urteilen). Demgegenüber steigt die Wahrscheinlichkeit der Verhängung einer lebenslangen Freiheitsstrafe in den Urteilen ab 2002 von vorher $12 \%$ auf $79 \%$. Diese Diskrepanz hinsichtlich der lebenslangen Freiheitsstrafen bei den Partnertötungsgrenzfällen ist auch der Hauptgrund für den starken Interaktionseffekt zwischen den Variablen »Stichprobe« und »Urteilsjahr«. Dieser Befund verdeutlicht erneut den Trend zu höheren Strafen bei den Ehrenmorden im Gegensatz zu den gleichbleibenden Strafhöhen in den Partnertötungsfällen. 
Abbildung 2 Modell-basierte Wahrscheinlichkeiten der Strafmaße in der Partnertötungsstichprobe und bei den Partnertötungsgrenzfällen der Ehrenmordstichprobe nach Urteilszeitraum

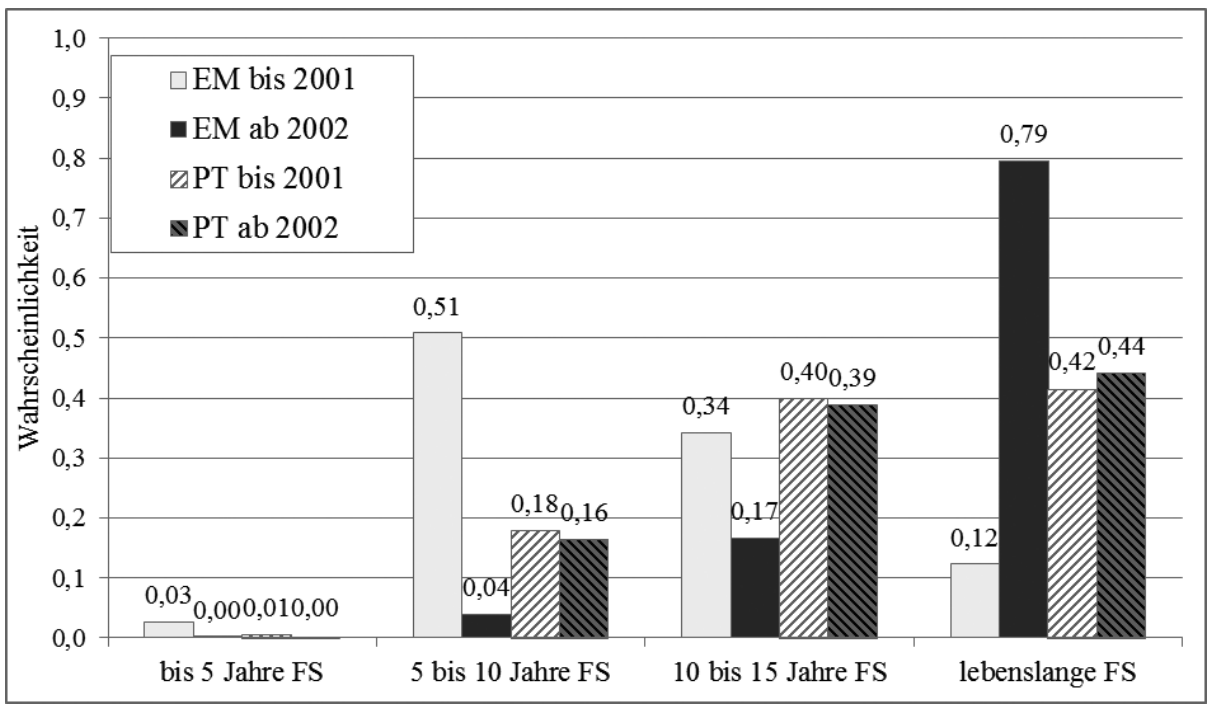

$\mathrm{N}=114$ Verurteilungen (91 Partnertötungen, 23 Grenzfälle zur Partnertötung)

$\mathrm{EM}=$ Partnertötungsgrenzfälle in der Ehrenmordstichprobe; PT = Partnertötungsfälle in der Vergleichsstichprobe

Warum die Partnertötungsgrenzfälle der Ehrenmordstichprobe in den Jahren bis 2001 milder beurteilt wurden als die Partnertötungen der Vergleichsstichprobe, kann nur vermutet werden. Möglicherweise wurde die Sozialisation der Täter, die aus Heimatländern mit sehr patriarchalen Wertanschauungen und Rollenbildern stammen, strafmildernd berücksichtigt, während das patriarchale (Besitz-)Denken von Tätern, die in Deutschland sozialisiert wurden, als verwerflicher bewertet wurde.

Bedenklich erscheint ein weiterer Befund der Studie: Für die überwiegende Zahl der von den Kammern in den Strafzumessungsbegründungen angeführten Aspekte wurde bei den bivariaten Analysen in beiden Stichproben kein signifikanter Zusammenhang mit den untersuchten abhängigen Strafmaßvariablen gefunden. Als problematisch ist dabei insbesondere der fehlende strafmildernde Einfluss eines Geständnisses des Täters zu bewerten. Die Befunde legen nahe, dass es sich bei dem In-Aussicht-Stellen von Strafmilderungen für Geständnisse und der Erwähnung der geständigen Einlassung des Täters in den Strafzumessungsgründen nur um eine Taktik handelt, um die Geständnisbereitschaft von Tätern zu erhöhen und dadurch die Beweislage zu verbessern. ${ }^{11}$ Dies erscheint im Hinblick auf das Recht des Täters zu schweigen und sich nicht selbst zu belasten, fragwürdig.

11 Auch Streng stellte in seiner Untersuchung zu Absprachen und Strafzumessung im Strafverfahren wegen Körperverletzung sowie wegen Sexualdelikten auf der multivariaten Ebene keinen strafmildernden Einfluss des Geständnisses fest (vgl. Streng 2006, S. 463 f.). Der fehlende Effekt von geständigen Einlassungen ist somit wohl nicht als tötungsdeliktsspezifisches Phänomen einzuordnen. Vielmehr deuten diese Befunde auf eine generelle Taktik der Strafgerichte zur Gewinnung von Geständnissen hin. 
Optimistisch im Hinblick auf die gebotene Objektivität der Strafrichter stimmen hingegen die Ergebnisse bezüglich des Einflusses der Variablen »Ethnie des Täters «, »Staatsangehörigkeit des Täters« und »Geburtsland des Täters«: Für keinen dieser Aspekte wurde ein signifikanter Effekt auf das in den Stichproben verhängte Strafmaß gefunden. Eine Diskriminierung oder Bevorzugung von ausländischen Angeklagten findet vor deutschen Schwurgerichten demnach weder in Ehrenmord- noch in Partnertötungsfällen statt.

\section{Zusammenfassung und Ausblick}

Die Analysen haben gezeigt, dass die deutschen Schwurkammern Ehrenmorde im untersuchten Zeitraum 1996-2005 sehr unterschiedlich bewerteten. In $28 \%$ der 50 Fälle, die als Tötungsdelikt eingeordnet wurden und bei denen die Ehre als Beweggrund bewertet wurde, haben die Richter das Ehrmotiv gemäß der aktuellen Rechtsprechung des BGH als niedrigen Beweggrund i.S.v. \$211 StGB eingeordnet und die Täter dementsprechend wegen Mordes zu lebenslanger Haft verurteilt. In 20 \% der Fälle wendeten die Strafkammern hingegen wegen der starken Verhaftung des Täters in den heimatlichen Wertvorstellungen die Ausnahmeregelung des BGH an und entschieden deshalb auf eine Verurteilung wegen Totschlags i.S.v. $\$ 212$ StGB. Bei 14 \% der Täter wurde das Vorliegen eines Ehrmotivs entsprechend der früheren Rechtsprechung des BGH auf objektiver Ebene abgelehnt. Bei 26 \% der Täter wurde der kulturelle Hintergrund der Tat zudem strafmildernd berücksichtigt. Diese Fälle sowie die Urteile, in denen das Ehrmotiv nicht als Mordmerkmal bewertet wurde, stammen allerdings weit überwiegend aus dem Zeitraum vor 2002. In den jüngeren Ehrenmordurteilen ab 2002 zeigt sich ein signifikanter Trend zu mehr lebenslangen sowie höheren zeitigen Freiheitsstrafen. Dieser ist mit hoher Wahrscheinlichkeit auf das BGH-Urteil vom Februar 2002 zurückzuführen, in welchem die bereits 1994 vom 2. Strafsenat eingeleitete, aber 1997 durch den 4. Strafsenat konterkarierte aktuelle Rechtsprechungslinie bekräftigt wurde, wonach das Ehrmotiv grundsätzlich als niedriger Beweggrund zu bewerten ist. Die Änderung der Bewertung der Ehrenmorde durch den BGH schlägt sich somit in der Rechtsprechung der Landgerichte nieder. Regionale Unterschiede in der Beurteilung von Ehrenmorden konnten nicht nachgewiesen werden.

Die Partnertötungen wurden insgesamt etwas milder beurteilt als die Ehrenmordfälle, was vorrangig, aber nicht ausschließlich auf die erwartungsgemäß höhere Zahl von vermindert schuldfähigen Tätern zurückzuführen ist. Interessanterweise wurden Grenzfälle zur Partnertötung bis zum Jahr 2001 milder bestraft als die vergleichend untersuchten Partnertötungen. Erst die punitive Trendwende bei den Ehrenmorden führte zu höheren Strafen in der Teilstichprobe der Partnertötungsgrenzfälle im Vergleich zu den Partnertötungen aus der Vergleichsstichprobe. Diese Partnertötungen wurden über den gesamten Untersuchungszeitraum gleichmäßig sanktioniert. Hier fanden sich keine Hinweise auf einen Anstieg der Strafhöhen.

Parallelen zwischen den Partnertötungs- und den Ehrenmordurteilen wurden insbesondere hinsichtlich der Faktoren festgestellt, die das Strafmaß beeinflussten. In beiden Stichproben waren die Anzahl der Todesopfer, die Schuldfähigkeit des Täters, die Tatplanung und -vorbereitung sowie das Vorliegen einer konfliktverschärfenden Vorszene von Bedeutung für die Höhe des Strafmaßes. Zudem wurden in keiner der beiden Stichproben Belege für einen strafmildernden Einfluss des Geständnisses sowie der überwiegenden Zahl der sonstigen in den Strafzumessungsbegründungen genannten Aspekte gefunden.

Die bivariaten und multivariaten Befunde der Studie sind aufgrund der recht kleinen Fallzahlen mit Bedacht zu bewerten, insbesondere wenn eine Auswertung nach Ehrenmordtypus vorgenommen wurde und dadurch sehr kleine Subgruppen entstanden. Da es sich bei den untersuchten Ehrenmorden allerdings - im Gegensatz zu den Partnertötungsfällen - 
nicht um eine Stichprobe im statistischen Sinne handelt, sondern aufgrund des angestrebten Ziels einer Vollerhebung zumindest ein sehr großer Teil der im Untersuchungszeitraum in Deutschland polizeilich bekannt gewordenen Ehrenmordfälle analysiert werden konnte, entfalten die Ergebnisse trotz der kleinen Fallzahlen eine große Aussagekraft. Statistisch robustere Ergebnisse könnten aufgrund der insgesamt geringen Anzahl von Ehrenmorden in Deutschland nur durch eine deutliche Ausweitung des Untersuchungszeitraumes erreicht werden. Eine solche Ausdehnung der Analysen auf jüngere Urteile wäre vor allem auch zur weitergehenden Überprüfung des festgestellten Anstiegs der Strafhöhen aufschlussreich. Die Befunde legen die Vermutung nahe, dass sich der Trend zu höheren Strafen bei der Ahndung der Taten seit 2005/2006 noch verstärkt hat. Dafür spricht zum einen die Bekräftigung der 1994 eingeleiteten Rechtsprechungslinie in weiteren BGH-Entscheidungen. Zum anderen ergibt sich ein solcher Eindruck aus der Medienberichterstattung über Ehrenmordprozesse in den letzten Jahren. In diesen Verfahren wurden hohe, häufig lebenslange Freiheitsstrafen verhängt. ${ }^{12}$

Wertvoll wäre außerdem eine weiterführende Untersuchung der strafrechtlichen Bewertung von Ehrenmorden durch die deutsche Justiz mittels Gruppendiskussionen sowie qualitativen Interviews mit Strafrichtern und idealerweise auch Staatsanwälten und Verteidigern, die in Ehrenmordprozessen mitgewirkt haben. Mithilfe dieser Erhebungsmethoden könnten Rückschlüsse auf die Gründe bestimmter Entscheidungen gezogen werden, über die hier nur Mutmaßungen angestellt werden konnten. Auf diesem Wege könnten auch weitere Erkenntnisse hinsichtlich der Ehrenmordfälle und des Prozessablaufs gewonnen werden, die aus den hier untersuchten Strafakten nicht hervorgehen. Zudem würden, insbesondere im Rahmen von Gruppendiskussionen, vermutlich auch die Einstellungen der Richter im Hinblick auf Themen wie die Integrationsdebatte deutlich werden, die für die Bewertung der Ehrenmorde eine bedeutende Rolle spielen.

\section{Literatur}

Agel, C. (2013). (Ehren-)Mord in Deutschland. Eine empirische Untersuchung zu Phänomenologie und Ursachen von »Ehrenmorden" sowie deren Erledigung durch die Justiz. Lengerich.

Backhaus, K., Erichson, B., Plinke, W. E Weiber, R. (2008). Multivariate Analysemethoden. Eine anwendungsorientierte Einführung. 12. Aufl. Berlin.

Baumeister, W. (2007). Ehrenmorde. Blutrache und ähnliche Delinquenz in der Praxis bundesdeutscher Strafjustiz. Münster.

Burmeister, J. (2011). Die schuldangemessene Bewertung von Ehrenmorden im deutschen Strafrecht. Differenzierte Schuldmerkmale auf Tatbestandsebene bei sonstigen niedrigen Beweggründen. Frankfurt a.M., Hamburg.

Çakir-Ceylan, E. (2011). Gewalt im Namen der Ehre. Eine Untersuchung über Gewalttaten in Deutschland und in der Türkei. Frankfurt a.M.

Cöster, A.C. (2009). Ehrenmord in Deutschland. Marburg.

Darnstädt, T. E Stoldt, H.-U. (2009). Folter gedeiht im Dunkeln. Interview mit Winfried Hassemer, Spiegel Online vom 13.05.2009; http://www.spiegel.de/politik/deutschland/0,1518,624304,00.html [28.05.2014].

Die Bundesregierung (2009). »Die Aussagen haben mich schockiert«. Staatsministerin Maria Böhmer kritisiert Äußerungen Winfried Hassemers zu Ehrenmorden, 14.05.2009; http://www.pressrelations.de/ new/standard/result_main.cfm?r=368110\&aktion=jour_pm [28.05.2014].

Deiß, M. E Goll, J. (2011). Ehrenmord. Ein deutsches Schicksal. Hamburg.

12 So beispielsweise im Fall der Tötung von Morsal O. (vgl. Hans 2009) sowie von Gülsüm S. (vgl. die Meldung bei Spiegel Online vom 30.12.2009; http://www.spiegel.de/panorama/justiz/vater-undbruder-verurteilt-lange-haftstrafen-im-ehrenmordfall-guelsuem-s-a-669382.html [28.05.2014]). 
Dölling, D. (1984). Probleme der Aktenanalyse in der Kriminologie, in: Kury, H. (Hrsg.), Methodologische Probleme in der kriminologischen Forschungspraxis. Köln, 265-286.

Elster, J. (1990). Norms of Revenge. Ethics 100/4, 862-885.

Erbil, B. (2008). Toleranz für Ehrenmörder? Soziokulturelle Motive im Strafrecht unter besonderer Berücksichtigung des türkischen Ehrbegriffs. Berlin.

Hans, B. (2009). Morsal-Prozess: »Was ist das, Ehre? Ich kenn keine Ehre!«. Spiegel Online vom 13.02.2009; http://www.spiegel.de/panorama/justiz/morsal-prozess-was-ist-das-ehre-ich-kenn-keine-ehre-a-607444.html [28.05.2014].

Kaspersson, M. (2009). «You Always Hurt the One You Love“: Homicide in a Domestic Context, in: Loucks, N., Smith Holt, S. \& Adler, J.R. (eds.), Why We Kill. Understanding Violence Across Cultures and Disciplines. London, 7-40.

Khan, R.A.A. (2012). Honour Killing. Roots and Remedies: A global view. New Delhi.

Kübnel, S.M. E Krebs, D. (2010). Multinomiale und ordinale Regression, in: Wolf, C. \& Best, H. (Hrsg.), Handbuch der sozialwissenschaftlichen Datenanalyse. Wiesbaden, 855-886.

Nisbett, R.E. E Cohen, D. (1996). Culture of Honor. The Psychology of Violence in the South. Boulder/CO.

Oberwittler, D. E Kasselt, J. (2011). Ehrenmorde in Deutschland 1996-2005. Eine Untersuchung auf der Basis von Prozessakten. Köln.

Oberwittler, D. E Kasselt, J. (2014). Honor Killings, in: Gartner, R. \& McCarthy, B. (eds.), Oxford Handbook of Gender, Sex and Crime. New York, Oxford, 652-670.

Saliger, F. (2003). Anmerkung zu BGH, Urteil vom 20.02.2002, 5 StR 538/01 (LG Bremen). Strafverteidiger 23, 21-25.

Schiffaner, W. (2008). Parallelgesellschaften. Wie viel Wertekonsens braucht unsere Gesellschaft? Für eine kluge Politik der Differenz. Bielefeld.

Sessar, K. (1980). Die Umgehung der lebenslangen Freiheitsstrafe. Monatsschrift für Kriminologie und Strafrechtsreform 63/4, 193-206.

Singer, K.H. (1994). Alttestamentarische Blutrachepraxis im Vergleich mit der Ausübung der Blutrache in der Türkei. Ein kultur- und rechtshistorischer Vergleich. Frankfurt a.M.

Streng, F. (2006). Verfahrensabsprachen und Strafzumessung. Zugleich ein empirischer Beitrag zur Strafzumessung bei Delikten gegen die Person, in: Feltes, T. u.a. (Hrsg.), Kriminalpolitik und ihre wissenschaftlichen Grundlagen. Festschrift für Professor Dr. Hans-Dieter Schwind zum 70. Geburtstag. Heidelberg, 447-467.

Streng, F. (2011). Forschungen zu Grundlagen und Determinanten der Strafzumessung, in: Frisch, W. (Hrsg.), Grundfragen des Strafzumessungsrechts aus deutscher und japanischer Sicht. Tübingen, 39-64.

Tellenbach, S. (2003). Ehrenmorde in der arabischen Welt. Anmerkungen zu Jordanien und anderen Ländern, in: Faath, S.H. (Hrsg.), Kriminalität in Nordafrika. Hamburg, 74-89.

TERRE DES FEMMES (2009). Keine falsche Toleranz für »Ehrenmörder! TDF-Stellungnahme zur Strafminderungsidee, 20.05.2009; http://frauenrechte.de/online/index.php/presse/pressearchiv/107pressearchiv2009/99-20052009-keine-falsche-toleranz-fuer-qehrenmoerderq-tdf-stellungnahme-zurstrafminderungsidee- [28.05.2014].

Valerius, B. (2011). Kultur und Strafrecht. Die Berücksichtigung kultureller Wertvorstellungen in der deutschen Strafrechtsdogmatik. Berlin.

(Anschr. d. Verf.: Dr. Julia Kasselt und PD Dr. Dietrich Oberwittler, Max-Planck-Institut für ausländisches und internationales Strafrecht, Günterstalstr. 73, 79100 Freiburg; julia.kasselt@gmx.de; d.oberwittler@mpicc.de) 